\title{
ARTICLE
}

\section{Dose rate produced by secondary radiation at the SwissFEL free electron laser facility}

\author{
Eike Hohmann*, Sven Reiche, Albert Fuchs, Romain Ganter, Frederic Le Pimpec, Roland Lüscher, Sabine Mayer and \\ Thomas Schietinger
}

Paul Scherrer Institute, 5232 Villigen - PSI, Switzerland

\begin{abstract}
A new large-scale facility is currently in the design phase at the Paul Scherrer Institute. SwissFEL is foreseen to accelerate electrons up to an energy of $7 \mathrm{GeV}$ with a pulsed time structure. Unavoidable interactions of the primary beam with beam line components create secondary radiation. The shielding of the accelerator vault has to reduce the radiation level for accessible areas in compliance with legal constraints. In addition, the position and layout of cable ducts and infrastructure shafts through the shielding of the accelerator tunnel have to be investigated from a radiation protection point of view. In this work, the dose rate distribution inside the accelerator vault has been evaluated for expected beam loss scenarios (normal and diagnostic mode of operation) based on Monte Carlo calculations. By means of the multi-particle transport code FLUKA, a simplified model has been defined and the results have been parameterized. From these, a dose rate evaluation has been extracted for different positions inside the accelerator tunnel. The results can be used to investigate the leakage of radiation through cable ducts and infrastructure shafts for different design layouts or modes of operation.
\end{abstract}

\section{Keywords: high-energy accelerator; free electron laser; Monte Carlo simulation; radiation protection}

\section{Introduction}

Designing the SwissFEL free electron laser facility is an iterative process subject to frequent changes affecting the layout of the building and its infrastructure. The actual layout of the accelerator, its shielding and the position and layout of ducts through it for infrastructure needs have to be investigated from a radiation protection point of view. For the SwissFEL project, this has been done in three stages. In a first step, beam losses have been evaluated by accelerator physicists based on the actual layout, experience and beam dynamic simulations - for normal operation, as well as for defined beam loss scenarios during diagnostic operation. From these, the dose rate inside of the accelerator vault arising from secondarily produced neutrons and photons has been calculated (stage two). Required ducts through the shielding can be investigated on the basis of the calculated dose rates according to their possible position, layout and design (stage three). Results are then compared to the dose rate constrains defined by the Swiss radiation protection ordinance [1].

The approach to independently investigate the dose rate inside the tunnel and the dose rate leaking through ducts was motivated by the frequent design changes (for instance shaft positions, accelerator layout). This procedure allows a prompt evaluation of the new situation, necessary in such a design phase. The method used for the first two stages is described in this work. The results are presented with reference to the current machine design (Figure 1). Details about the project and the accelerator layout can be found in the conceptual design report (CDR) [2].

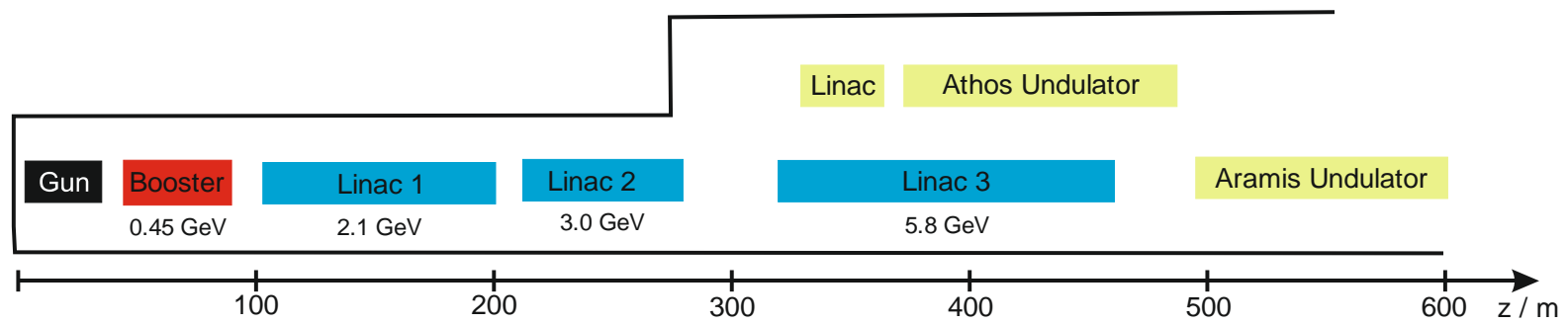

Figure 1. Schematic layout of the SwissFEL facility according to the CDR [2]. The data refers to the position along the beam line defined as $z$.

*Corresponding author. Email: Eike.Hohmann@psi.ch 


\section{Dose rate due to secondary radiation}

To estimate the ambient dose equivalent rate (subsequently referred to as dose rate) due to the secondary radiation from interactions of the primary beam with beam line components, a suitable set of data is mandatory. Since copper is a widely used material for beam line components, the dose rate arising from electrons impinging on a cylindrical copper target is adequate for this purpose. The dose rate due to neutrons and photons arising from electrons impinging on a copper target have been estimated using the multi-purpose Monte Carlo code FLUKA [3,4]. For this purpose, FLUKA calculations have been carried out for electron energies $E$ from $30 \mathrm{MeV}$ to $8 \mathrm{GeV}$ and target thicknesses $d$ from $2.5 \mathrm{~cm}$ to $45 \mathrm{~cm}$. The dose rate $D$ is scored in spherical volumes at a distance of $1 \mathrm{~m}$ to the target for angles $\alpha$ from $0^{\circ}$ to $180^{\circ}$ with respect to the beam axis (Figure 2).

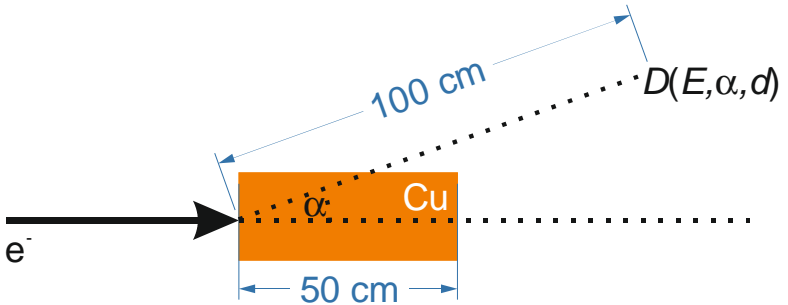

Figure 2. Model used to estimate the dose rate due to secondary radiation.
Due to the large number of data points, a parameterization of the results has been applied. The dose rate in a distance $r$ to the beam line can be evaluated through

$$
D(E, \alpha, d, r)=\exp \left(a_{0} \ln (E)+a_{1}\right) \cdot D_{1 m}^{0}(\alpha, d) \cdot\left(\frac{1 m}{r}\right)^{2}
$$

using the parameters summarized in Table 1. The estimates of the mean dose rate $D_{1 m}^{0}(\alpha, d)$ in dependency of the target thickness have been parameterized with a spline interpolation using routines from [5] (exemplarily shown in Figure 3).

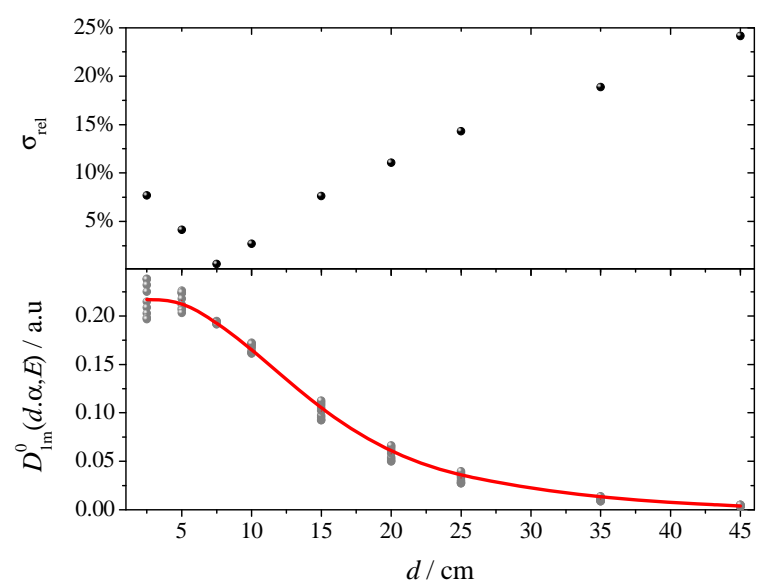

Figure 3. The FLUKA estimates of the mean dose rate in dependency of the target thickness (gray, bottom) with standard deviation (top) have been interpolated using a spline interpolation (red)[5] - exemplary data.

Table 1. Normalized angle dependent dose rate in pSv per electron with corresponding scaling parameter as defined in eq 1.

\begin{tabular}{|c|c|c|c|c|c|c|c|c|c|c|}
\hline $\mathrm{r} / \mathrm{cm}$ & $0^{\circ}$ & $20^{\circ}$ & $40^{\circ}$ & $60^{\circ}$ & $80^{\circ}$ & $100^{\circ}$ & $120^{\circ}$ & $140^{\circ}$ & $160^{\circ}$ & $180^{\circ}$ \\
\hline \multicolumn{11}{|c|}{ Neutron data } \\
\hline 2.5 & $5.10 \mathrm{E}-2$ & 3.56E-01 & 2.72E-01 & 2.27E-01 & 2.14E-01 & $1.76 \mathrm{E}-01$ & 1.13E-01 & 7.91E-02 & 5.63E-01 & 4.70E-02 \\
\hline 5.0 & $1.16 \mathrm{E}-2$ & 2.11E-01 & $2.28 \mathrm{E}-01$ & 2.19E-01 & $2.16 \mathrm{E}-01$ & $1.78 \mathrm{E}-01$ & 1.17E-01 & 8.79E-02 & 7.48E-02 & $7.09 \mathrm{E}-02$ \\
\hline 7.5 & $1.41 \mathrm{E}-2$ & $1.42 \mathrm{E}-01$ & 1.83E-01 & $1.92 \mathrm{E}-01$ & $1.94 \mathrm{E}-01$ & 1.65E-01 & 1.19E-01 & $1.00 \mathrm{E}-01$ & 9.32E-02 & $9.30 \mathrm{E}-02$ \\
\hline 10.0 & $3.18 \mathrm{E}-2$ & $1.06 \mathrm{E}-01$ & $1.48 \mathrm{E}-01$ & 1.63E-01 & $1.68 \mathrm{E}-01$ & $1.49 \mathrm{E}-01$ & $1.21 \mathrm{E}-01$ & $1.12 \mathrm{E}-01$ & $1.10 \mathrm{E}-01$ & $1.10 \mathrm{E}-01$ \\
\hline 15.0 & $9.28 \mathrm{E}-2$ & $5.46 \mathrm{E}-02$ & 8.29E-02 & 9.84E-02 & $1.02 \mathrm{E}-01$ & $1.06 \mathrm{E}-01$ & $1.15 \mathrm{E}-01$ & $1.22 \mathrm{E}-01$ & 1.27E-01 & $1.29 \mathrm{E}-01$ \\
\hline 20.0 & $1.53 \mathrm{E}-1$ & 3.52E-02 & 4.50E-02 & 5.54E-02 & 5.80E-02 & 7.66E-02 & $1.08 \mathrm{E}-01$ & $1.24 \mathrm{E}-01$ & 1.32E-01 & $1.35 \mathrm{E}-01$ \\
\hline 25.0 & $1.91 \mathrm{E}-1$ & 3.05E-02 & $2.51 \mathrm{E}-02$ & 3.13E-02 & 3.24E-02 & 6.03E-02 & $1.04 \mathrm{E}-01$ & $1.25 \mathrm{E}-01$ & 1.34E-01 & 1.37E-01 \\
\hline 35.0 & $2.24 \mathrm{E}-1$ & $1.16 \mathrm{E}-02$ & $9.78 \mathrm{E}-03$ & 1.03E-02 & $1.05 \mathrm{E}-02$ & 4.68E-02 & $1.01 \mathrm{E}-01$ & $1.25 \mathrm{E}-01$ & $1.36 \mathrm{E}-01$ & $1.39 \mathrm{E}-01$ \\
\hline 45.0 & $2.30 \mathrm{E}-1$ & 3.39E-02 & 6.03E-03 & 3.57E-03 & 3.67E-03 & 4.30E-02 & $1.00 \mathrm{E}-01$ & $1.25 \mathrm{E}-01$ & $1.36 \mathrm{E}-01$ & $1.39 \mathrm{E}-01$ \\
\hline $\mathrm{a} 0$ & 1.43 & 1.22 & 1.18 & 1.16 & 1.14 & 1.08 & 1.00 & 0.98 & 0.97 & 0.96 \\
\hline a1 & -8.63 & -6.98 & -6.14 & -5.84 & -5.79 & -5.71 & -5.42 & -5.25 & -5.18 & -5.17 \\
\hline \multicolumn{11}{|c|}{ Photon data } \\
\hline 2.5 & 6.73E-01 & 8.28E-01 & 6.55E-01 & 5.82E-01 & 5.70E-01 & $5.10 \mathrm{E}-01$ & 3.65E-01 & 2.35E-01 & $1.32 \mathrm{E}-01$ & $9.25 \mathrm{E}-02$ \\
\hline 5.0 & 3.73E-02 & 1.38E-01 & 2.29E-01 & 2.53E-01 & 2.58E-01 & 2.21E-01 & $1.62 \mathrm{E}-01$ & $1.28 \mathrm{E}-01$ & $1.14 \mathrm{E}-01$ & $1.09 \mathrm{E}-01$ \\
\hline 7.5 & 3.54E-02 & 2.71E-02 & 8.15E-02 & $1.06 \mathrm{E}-01$ & 1.09E-01 & $9.98 \mathrm{E}-02$ & $9.60 \mathrm{E}-02$ & $1.01 \mathrm{E}-01$ & $1.06 \mathrm{E}-01$ & $1.11 \mathrm{E}-01$ \\
\hline 10.0 & 4.07E-02 & 6.19E-03 & 3.01E-02 & 4.63E-02 & 4.92E-02 & 5.39E-02 & 7.32E-02 & $9.24 \mathrm{E}-02$ & 1.09E-01 & $1.14 \mathrm{E}-01$ \\
\hline 15.0 & 4.24E-02 & 5.33E-04 & 4.34E-03 & $9.14 \mathrm{E}-03$ & $1.05 \mathrm{E}-02$ & 2.73E-02 & $6.20 \mathrm{E}-02$ & 9.01E-02 & $1.08 \mathrm{E}-01$ & $1.16 \mathrm{E}-01$ \\
\hline 20.0 & 4.34E-02 & $1.85 \mathrm{E}-04$ & 6.87E-04 & $1.98 \mathrm{E}-03$ & 2.47E-03 & 2.28E-02 & 6.05E-02 & 8.89E-02 & $1.08 \mathrm{E}-01$ & $1.14 \mathrm{E}-01$ \\
\hline 25.0 & 4.21E-02 & 2.05E-04 & 1.03E-04 & 4.72E-04 & $6.00 \mathrm{E}-04$ & 2.18E-02 & 6.06E-02 & 8.89E-02 & $1.08 \mathrm{E}-01$ & $1.14 \mathrm{E}-01$ \\
\hline 35.0 & 4.30E-02 & $1.84 \mathrm{E}-04$ & 2.33E-05 & 4.23E-05 & 5.84E-05 & 2.16E-02 & $6.02 \mathrm{E}-02$ & 8.82E-02 & $1.08 \mathrm{E}-01$ & $1.15 \mathrm{E}-01$ \\
\hline 45.0 & 4.26E-02 & $1.81 \mathrm{E}-04$ & $2.25 \mathrm{E}-05$ & $1.08 \mathrm{E}-05$ & $1.92 \mathrm{E}-05$ & $2.16 \mathrm{E}-02$ & 6.05E-02 & 8.81E-02 & 1.07E-01 & $1.14 \mathrm{E}-01$ \\
\hline $\mathrm{a} 0$ & 1.32 & 1.04 & 1.01 & 1.00 & 1.00 & 0.91 & 0.74 & 0.64 & 0.59 & 0.57 \\
\hline a1 & -8.73 & -4.65 & -4.71 & -5.12 & -5.55 & -5.88 & -5.99 & -6.06 & -6.11 & -6.11 \\
\hline
\end{tabular}




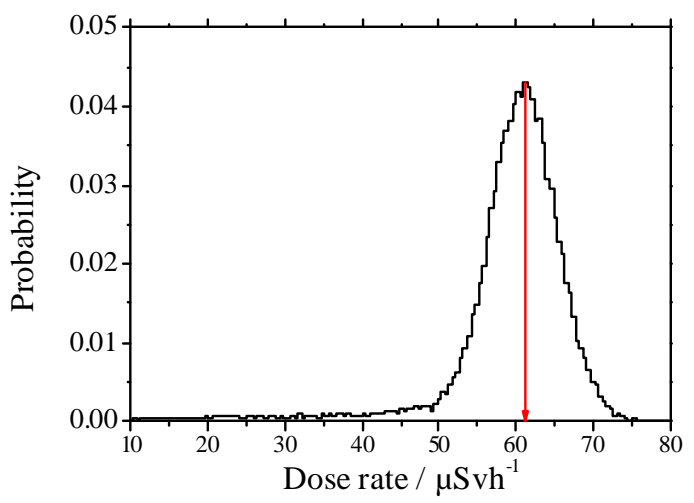

Figure 4. Probability distribution of the dose rate, taking into account the ambiguity due to the accelerator layout and the model. The given values and their uncertainties are derived through the first and second statistical moment.

The dose rates due to neutrons obtained by FLUKA and evaluated from the model deviate by less than $35 \%$ for electron energies greater $50 \mathrm{MeV}$ and angles $\alpha$ greater than $30^{\circ}$. From a radiation protection point of view, the dose rate in the forward direction is not of great interest. As a result, the length of the copper target of $50 \mathrm{~cm}$ used for the calculations ensures, that the high-energy bremsstrahlung component is stopped and therewith the secondary neutron production is maximized.

\section{Dose rate evaluation}

Dose rate estimates using the parameterization described above depend on ambiguous assumptions about local beam losses. These have been defined using an adequate target thickness, energy of the interacting electrons and position of the beam loss. To account for the uncertainty arising from these assumptions, the dose rate is calculated with varying the parameters by means of methods from [5] to obtain a probability distribution as shown in Figure 4. The mean value of the resulting dose rate with associated standard deviation is derived by means of the first and second statistical moment. Since the calculated does rates are used for radiation

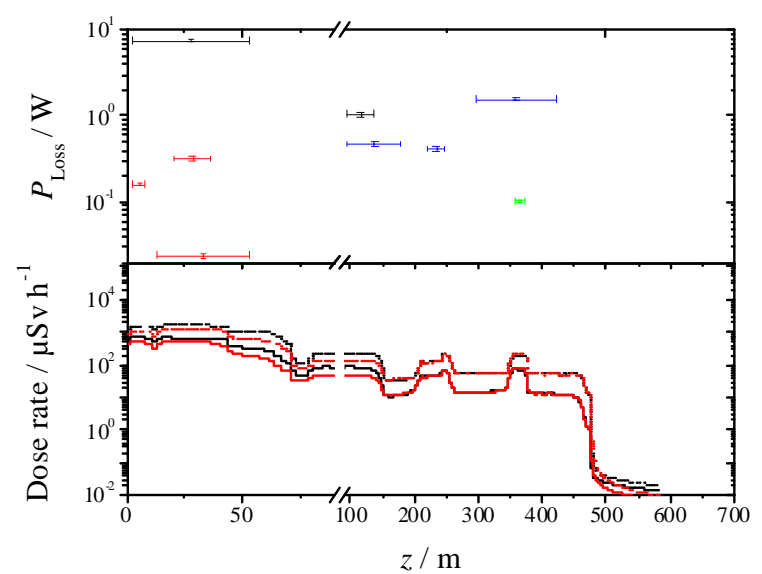

Figure 5. Dose rate from neutrons (black) and photons (red) in the accelerator vault, due to dark current losses from different sections defined in Figure 1 (upper part). The dotted lines indicate the highest expected values. protection purposes, a value increased by one standard deviation is used for subsequent investigations of ducts in the shielding.

\section{Expected dose rate due to secondary radiation at SwissFEL}

The thickness of the beam line component affects the dose arising from interactions of the primary beam with these targets. Due to the layout of the accelerator, target thickness of $(25.0 \pm 7.5) \mathrm{cm}$ is assumed in the gun and booster region, where many magnets and solenoids are foreseen, the target thickness is estimated with $(25.0 \pm 7.5) \mathrm{cm}$. In the linac region, where the main beam losses occur in the cavities, a wall thickness of $(10.0 \pm 3.0) \mathrm{cm}$ is used.

The expected secondary radiation can be characterized by its origin and be described by the two following scenarios.

\subsection{Normal operation}

During normal operation, the beam is optimized for small emittance to achieve the FEL-pulse requirements. This results in small losses, negligible from a radiation protection point of view.

Electrons produced spontaneously due to field emission from the photocathode and in the accelerating cavities (referred to as dark current) are propagated through the accelerator. Simulations have been carried out to investigate the properties of these electrons [6]. Since the accelerator is optimized for the FEL-pulse, the dark current not originating from the gun is lost near the next magnet and therefore has only energies of a few hundred $\mathrm{MeV}$. The calculated dose rates are based on conservative estimates of the position $z_{\mathrm{L}}$, origin of the loss point, the loss power $P_{\mathrm{L}}$ and of the mean energy $E_{\mathrm{L}}$ summarized in Table 2 [7].

Table 2. Conservative estimates of parameters used to calculate the dose rate arising due to dark current[7].

\begin{tabular}{llll}
\hline Origin & $z_{\mathrm{L}} / \mathrm{m}$ & $P_{\mathrm{L}} / \mathrm{W}$ & $E_{\mathrm{L}} / \mathrm{GeV}$ \\
\hline Gun & $28.0 \pm 25.0$ & $7.5 \pm 0.4$ & $0.15 \pm 0.05$ \\
& $116.0 \pm 20.0$ & $1.1 \pm 0.1$ & $0.35 \pm 0.11$ \\
Booster 1 & $5.5 \pm 2.5$ & $0.2 \pm 0.0$ & $0.04 \pm 0.01$ \\
& $33.0 \pm 20.0$ & $0.1 \pm 0.0$ & $0.12 \pm 0.04$ \\
Booster 2 & $28.7 \pm 8.3$ & $0.3 \pm 0.0$ & $0.04 \pm 0.01$ \\
Linac 1 & $137.7 \pm 41.7$ & $0.5 \pm 0.0$ & $0.07 \pm 0.02$ \\
Linac 2 & $234.4 \pm 13.7$ & $0.4 \pm 0.0$ & $0.13 \pm 0.04$ \\
Linac 3 & $359.1 \pm 63.7$ & $1.6 \pm 0.1$ & $0.13 \pm 0.04$ \\
Athos UL & $364.4 \pm 7.3$ & $0.1 \pm 0.0$ & $0.07 \pm 0.02$ \\
\hline
\end{tabular}

During normal operation, the highest dose rate due to secondary radiation is expected in the injector region with maximal values of $1.5 \mathrm{mSvh}^{-1}$. Figure 5 shows the expected dose rate distribution in the tunnel.

\subsection{Diagnostic operation}

For machine tuning and optimization, luminescent screens made of LuAlO with a thicknesses of $20 \mu \mathrm{m}$ or 
$200 \mu \mathrm{m}$ (referred to as type 0 and 1 , respectively) and Si with a thickness of $300 \mu \mathrm{m}$ (referred to as type 2) will be inserted in the beam line. This increases the emittance and leads to secondary radiation due to the interaction of the primary beam with beam line components further downstream. The beam losses have been calculated with FLUKA for discrete electron energies from $100 \mathrm{MeV}$ to $8 \mathrm{GeV}$. The results have been parmeterized with

$$
\frac{d \Phi}{d x} \propto \exp \left(-\frac{1}{2}\left(\frac{\log (x)-\log \left(a+b \ln (E)+c \ln (E)^{2}\right)}{0.216}\right)^{2}\right)
$$

and the set of parameters summarized in Table 3 . The deviation of the simulated results to the parameterized ones for the total electron loss is below $25 \%$ in the covered energy range.

Table 3. Parameters defined in equation 2 to evaluate the beam loss due the insertion of different screens.

\begin{tabular}{cccc}
\hline & Type 0 & Type 1 & Type 2 \\
\hline a & 8.27 & 7.07 & 7.91 \\
b & 0.96 & 0.87 & 0.93 \\
c & 0.03 & 0.04 & 0.04 \\
\hline
\end{tabular}

To validate the model, measurements have been carried out at the SwissFEL injector test facility with a $230 \mathrm{MeV}$ electron beam. Inserting a screen of type 1 into the primary beam leads to a beam loss with comparable shape (Figure 6). In diagnostic mode, the losses due to dark current can be neglected.

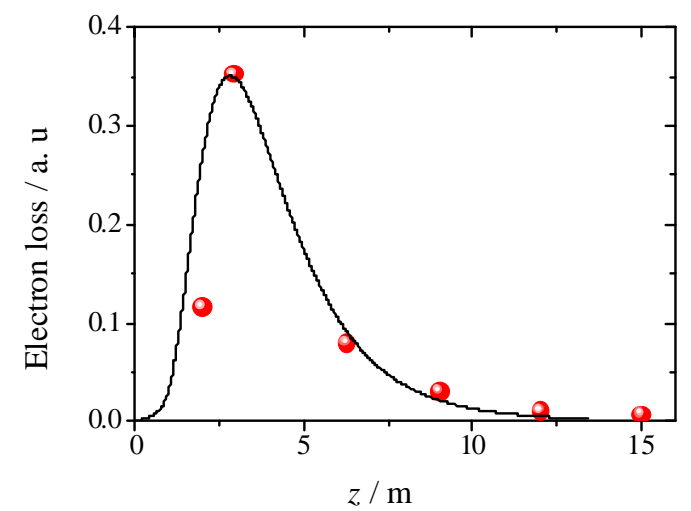

Figure 6. Comparison of the calculated (black) to the measured (red) beam loss for electrons with an energy of $230 \mathrm{MeV}$ and a screen of type 1 .

In this mode of operation, the electron current will be reduced to $200 \mathrm{pA}$. Since this mode of operation is not foreseen for long term usage, a duty cycle has be assigned to every screen (Figure 7, top) to scale the dose rate arising from its use.

The estimated dose rate due to neutrons (resp. photons) in a distance of $1 \mathrm{~m}$ to the beam line is lower than $500 \mu \mathrm{Svh}^{-1}\left(100 \mu \mathrm{Svh}^{-1}\right)$. It increases, depending on the energy of the primary beam, downstream of the position where the screen is inserted. Since the screens cannot be used simultaneously, the maximum dose rate due to neutrons and photons for each position is estimated for radiation protection purposes. The dose

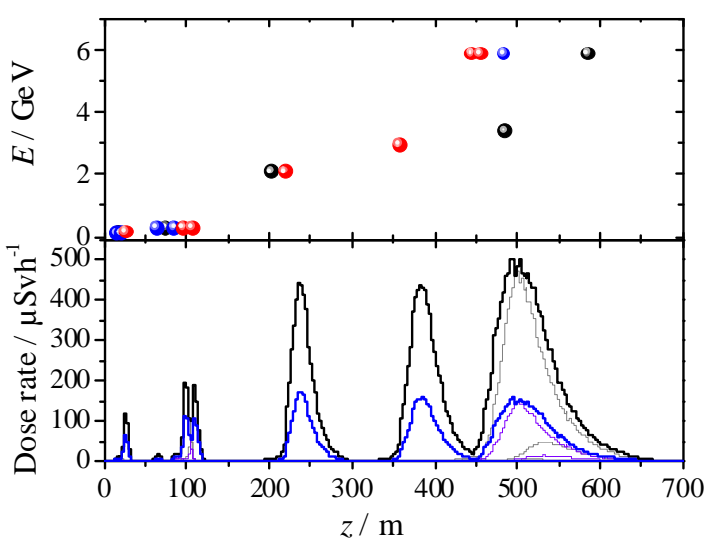

Figure 7. Maximum estimated dose rate distribution due to neutrons (black) and photons (blue) in a distance of $1 \mathrm{~m}$ in the accelerator tunnel using a screen type 1 (bottom). The screens have expected duty cycles of $1 \%$ (black), $10 \%$ (red) and $89 \%$ (blue) of the time, when the machine operates in diagnostic mode (top).

rate distribution in the accelerator tunnel is shown in Figure 7 (bottom).

\section{Conclusions}

Secondary radiation at the SwissFEL accelerator facility arises, during normal operation, mainly from dark current or, during diagnostic operation, from inserting diagnostic screens. The occurring dose rate due to neutrons and photons is distributed over the length of the accelerator and influences the position and layout of ducts through the shielding.

A flexible tool to estimate the dose rate at any point in the accelerator vault has been developed for radiation protection purposes. Being based on parameterized models, it allows reassessing the situation after a design change more rapidly than a full simulation of the tunnel would achieve. First measurements with a $230 \mathrm{MeV}$ electron beam lead to values comparable to the prediction from the model.

\section{References}

[1] Swiss Radiological Protection Ordinance (SR 814.501), (1994).

[2] R. Ganter (editor), SwissFEL Conceptual Design Report, http://www.psi.ch/swissfel/, (2012).

[3] G. Battistoni, S. Muraro, P.R. Sala, F. Cerutti, A. Ferrari, S. Roesler, A. Fasso and J. Ranft, The FLUKA code: Description and benchmarking, Proceedings of the Hadronic Shower Simulation Workshop 2006, AIP 896, (2007), pp. 31-49.

[4] V. Vlachoudis, FLAIR: A powerful but user friendly graphical interface for FLUKA, Int. Conf. on Mathematics, Proc. Computational Methods \& Reactor Physics, New York, (2009).

[5] W. H. Press, S.A. Teukolsky, W.T. Vettering and B.P. Flannery, Numerical Recipes in C (Second Edition), (1992). ISBN 0-521-4318-5

[6] F. Le Pimpec, R. Zennaro, S. Reiche, E. Hohmann, A. Citterio and A. Adelmann, Dark Current Studies 
for SwissFEL, http://arxiv.org/abs/1205.3098 (2012).
[7] S. Reiche, Conservative beam losses at SwissFEL private communication, (2012). 